\title{
Effect of Breast Cancer Molecular Subtypes on Survival in Patients with Liver Metastases
}

\author{
E. A. Elkhouly ${ }^{1}$, E. A. Tawfik ${ }^{1}$, T. M. Rageh², M. Dawoud ${ }^{3}$ and W. S. Morad ${ }^{4}$ \\ ${ }^{1}$ Department of Clinical Oncology, ${ }^{2}$ Departement of General Surgery, ${ }^{3}$ Department of Pathology, \\ Faculty of Medicine, ${ }^{4}$ Department of Environmental Health of Liver, National Liver Institute, Menoufia \\ University, Shebin El-Koom, Egypt
}

\begin{abstract}
Background: The liver is one of the most common metastatic sites of breast cancer, hepatic metastases develop in $6 \%-25 \%$ of patients with breast cancer and being associated with a poor prognosis. The aim of this study was to analyze the clinical characteristics and survival of patients with different molecular breast cancer subtypes who developed hepatic metastases (HM), and to investigate the prognostic and predictive factors that affect clinical outcome.

Methods: A retrospective study of 63 early breast cancer patients (stage $I \& \Pi$ ) who developed hepatic metastases, diagnosed at Menoufia University Hospital, Clinical Oncology Department from 1st of January 2000 to 31 of May 2007. Molecular Subtypes were defined as luminal A, luminal B, human epidermal growth factor receptor 2 (HER2) enriched, triple-negative (TN). Prognostic factors correlation with clinical features and treatment approaches were assessed after diagnosis of HM.

Results: Median Overall Survival (OS) of all breast cancer patients with HM is 35 months, with statistically significant median OS among breast cancer molecular subtypes: luminal A, luminal B, HER2 enriched and TN $(P=0.02)$, Median OS was 33.27, 45.75, 34.14, 30 months respectively. In multivariate analysis, the initial disease stage and the number of hepatic metastases are the independent predictors of prognosis. Luminal A group of patients who were treated with endocrine therapy didn't significantly improve more than patients treated without endocrine therapy when indicated (median survival of 35 vs. 30 months, $P=0.19$ ). Similarly there were no significant differences in Luminal B group $(P=0.13)$. However, a good tendency for better median survival for patients treated with endocrine therapy was found as median survival is 48 months Vs 40 months in patients not treated with endocrine therapy after metastases. Endocrine therapy was a significantly favorable prognostic factor for luminal subtype patients, who were initially treated with adjuvant endocrine therapy $(P=0.03)$.

Conclusions: Breast cancer molecular subtypes were associated with survival benefit difference after hepatic metastases. Endocrine therapy was a significantly favorable treatment for patients with luminal subtypes.
\end{abstract}

Key words: breast cancer, hepatic metastases, molecular subtypes, prognostic variables, survival analysis. Corresponding Author: Enas Abou Bakr Elkhouly E-mail: enasaboubaker6@hotmail.com

\section{INTRODUCTION}

Breast cancer is the most common cancer in women in the world ${ }^{1}$. Despite improvement in treatment, 20\%$30 \%$ of patients with early breast cancer will experience metastatic disease ${ }^{2}$. Meanwhile, $6 \%-10 \%$ of patients were to be ill with metastatic disease at the initial diagnosis of breast cancer ${ }^{3}$. Hepatic metastases are the one of the most frequent distant metastases of breast cancer ${ }^{1}$. Survival rate for patients with hepatic metastases is poor, with a median survival time about 14 months $^{4}$.

Breast cancer has different molecular subtypes which may be defined by gene expression profiles ${ }^{5}$ or immunohistochemical biomarkers ${ }^{6}$. It is reported that human epidermal growth factor receptor 2 (HER2)-enriched subtype breast cancers aggressively spread to the liver ${ }^{7}$. Endocrine therapy is indicated for estrogen receptor (ER) and/or progesterone receptor $(\mathrm{PgR})$ positive patients with long disease free survival, non life threatening metastatic sites (such disease should be non visceral or asymptomatic visceral disease), and good performance status. Major treatments for hepatic metastases from breast cancers include chemotherapy, surgery, and intervention therapy ${ }^{8}$.

The aim of this study is to analyze the clinical characteristics and survival of patients with different molecular breast cancer subtypes who developed HM, and to investigate the prognostic and predictive factors that affect clinical outcome. 


Vol. 10 |No. 1-2 $2014 \quad$ E. A. Elkhouly et al.

\section{PATIENTS AND METHODS}

Sixty three early breast cancer patients (stage I\&M) with no evidence of distant metastases at the time of primary diagnosis with breast cancer were followed up at clinical oncology department, Menoufia University from 1st of January 2000 to 31 of May 2007. It is taken into consideration that the initial metastatic site should be related to liver.

Clinical characteristics included personal information, pathologic subtype, disease stage, treatment, location and time of metastasis and ER, PgR and HER-2 expression. The TNM Cancer Staging Manual 7th edition of the American Joint Committee on Cancer (AJCC) ${ }^{9}$ was used to stage these patients. Breast cancers were classified into four molecular subtypes according to gene expression profile-validated immuno-histochemical surrogate panel as follows: luminal A (ER positive and/or PgR positive and HER-2 negative), luminal B (ER positive and/or PgR positive and HER-2 positive), HER-2 enriched (ER negative and PgR negative and HER-2 positive), and triple-negative (TN) (ER negative and PgR negative and HER-2 negative) ${ }^{10}$.

The concept of oligo-metastatic breast cancer (OMBC) is a new paradigm proposed by Hellman and Weichselbaum. It suggests that patients with oligometastases, either de novo or following systemic treatment could potentially be cured by ablation of these lesions; whereas more advanced metastatic disease will require more aggressive and effective systemic treatment ${ }^{11}$.

The term OMBC has now become synonymous with limited metastases, solitary metastasis, isolated metastases, or minimal metastases. However, OMBC is not yet sufficiently recognized. Some suggest that an aggressive approach with multidisciplinary treatment would be beneficial for potential long-term CR or even cure, whereas little evidence is available regarding outcomes of OMBC and their long-term prognosis ${ }^{12}$.

Another and more specific definition is that OMBC is identified as: one or 2 organs involved with metastatic lesions (excluding the primary lesion resectable by surgery), fewer than 5 lesions per metastasized organ, and lesion diameter less than $5 \mathrm{~cm}^{11}$.

Despite the fact that no randomized data exist to support any particular follow-up sequence or protocol, balancing patient needs and follow-up costs, it is recommended to make regular visits every 3 to 4 months in the first 2 years, every 6 months from years $3-5$ and annually thereafter. Every visit included thorough history taking, eliciting of symptoms and physical examination.
Ipsilateral (after BCS) and contralateral mammography is recommended every 1 to 2 years. An MRI of the breast may be indicated for young patients, especially in the case of dense breast tissue and genetic or familial predispositions. In asymptomatic patients, there are no data to indicate that other laboratory or imaging tests (e. g. blood counts, routine chemistry tests, chest X-rays, bone scans, liver ultrasound exams, CT scans or any tumor markers such as CA15-3 or CEA) produce a survival benefit ${ }^{13}$. Hepatic metastases were diagnosed by liver ultrasound, computed tomography or magnetic resonance imaging.

\section{Statistics}

All data was analyzed by SPSS version 21.0 software. Survival analysis was estimated using the Kaplan-Meier survival analysis curve including number of survived patients, median survival time at $95 \%$ confidence interval (CI). Survival was compared across subtypes using the log-rank test. Statistical comparisons were carried out using $\mathrm{T}$ test or analysis of variance (ANOVA) for quantitative variables and Pearson's Chi-squared test or Fisher's exact test for categorical variables. Multivariate analysis was estimated by creating a Cox proportional hazards regression model. P-value $<0.05$ was considered statistically significant.

\section{RESULTS}

Of the 63 patients included in the study, all had undergone a follow-up interval from initial diagnosis of breast cancer at 1 st of January 2000 to 31 of May 2007 with a median follow-up interval 24 months (2-76 months).

The age of the patients ranged from 30-66 years, with a median of 42 years. 39 cases $(61.90 \%)$ were pre-menopausal. 53 cases $(84.12 \%)$ were invasive ductal carcinoma, 6 cases $(9.52 \%)$ were invasive lobular carcinoma and 4 cases $(6.36 \%)$ were other types. The cancer stages were classified as follows: stage ,26 cases; stage ,37 cases. Among 63 cases, 33 $(52.38 \%)$ were luminal A, 8 (12.69\%) were luminal $\mathrm{B},{ }^{14}(22.24 \%)$ were HER-2 enriched, 8 (12.69\%) were TN.

Of the 63 patients included in the study, all had undergone a follow-up from initial diagnosis of breast cancer to May 31, 2007 with a median follow-up interval 24 months (2-76 months), with median interval from initial diagnosis to HM 24.68 months. OS (survival from diagnosis date till date of death or last follow up) among different subtypes was significantly different $(P=0.02)$, however the DFS (survival from the end of treatment till time of recurrence) was not statistically significant $(P=0.16)$ (Table 1). 
Univariate analysis for survival after hepatic metastases in all molecular subtypes showed the following parameters as significant prognostic factors: disease stage at presentation, molecular subtype, number of hepatic metastases (Table 2).

Multivariate analysis was performed including disease stage at presentation, molecular subtype, and number of hepatic metastases. Advanced stage, non luminal subtypes, and multiple HM were carried out to be unfavorable independent prognostic factors (Table 2).

Median OS of all breast cancer patients is 35 months while mean OS is 36.12 months (Figure 1).

Median DFS after first diagnosed with breast cancer was 18.24 months (3-42 months). The one-, twothree-, four-, five-year survival rates after breast cancer diagnosis were $63.5 \%, 31.7 \%, 15.6 \%, 10.8 \%$, and $5.4 \%$, respectively (Figure 2).

The median survival before HM among breast cancer subtypes: luminal A, luminal B, HER2 enriched and TN was significant $(P=0.03)$, with median survival was 34 , 48, 36, 32 months respectively. Median survival after HM were 12 months (luminal A), 24 months (luminal B), 20 months (HER2-enriched), and 17 months (TN), with a significant difference $(P=0.01)$.

There is a statistical significant difference $(P=0.007)$ between median OS of disease stage 2 and 3 which are 40 and 33 months respectively (figure 3 ).

The median OS of patients according to treatment with hormonal therapy after metastases: TAM, AI, or no hormonal therapy is statistically significant $(P=0.046)$ which are 48,48 , and 33 months respectively (figure 4 ).

The median OS between isolated and multiple hepatic metastases is statistically significant $(P=0.001)$ which are 40, and 32 months respectively (figure 5).

The median survival of patients with liver only metastases and those with multiple metastatic sites including liver is 36 and 32 months respectively, and this is not statistically significant $(P=0.82)$ (figure 6).

In luminal A group of patients, endocrine therapy didn't significantly affect the outcome of patients treated versus no endocrine therapy (median survival of $35 \mathrm{vs}$. 30 months, $P=0.19$, (Figure 7 ).
There were no significant survival differences in luminal B group treated by hormonal therapy after HM $(P=0.13)$, (Figure 8), However, a better median survival was found for patients treated with endocrine therapy (48 months) versus 40 months more than patients who were not treated with endocrine therapy after HM, which is in general, better than luminal A. HER2-enriched group ( $P=$ Not applicable as there is no cases treated with targeted hormonal therapy after metastases).

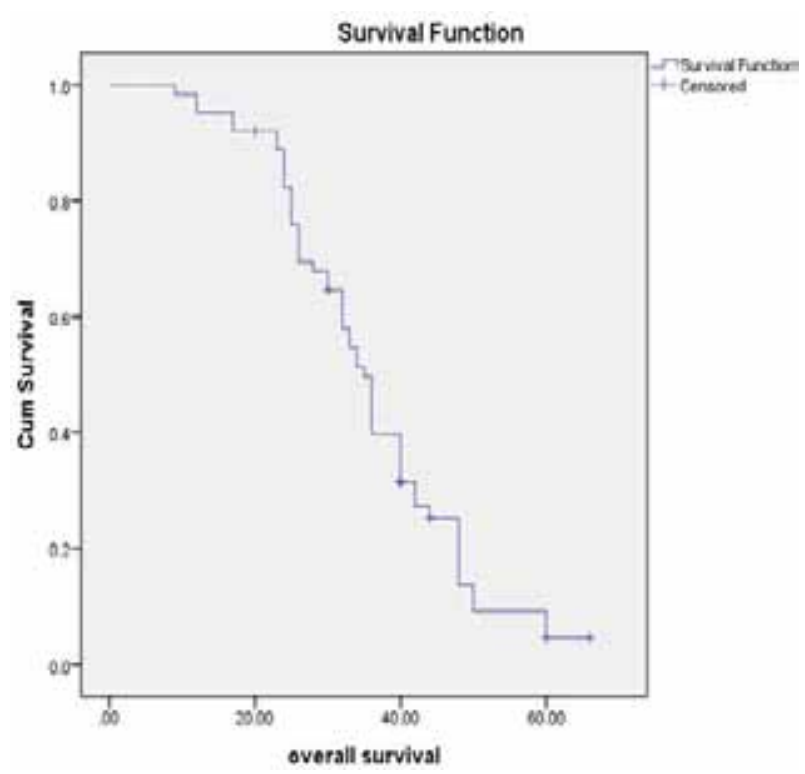

Figure 1: OS for all patients after HM from breast cancer.

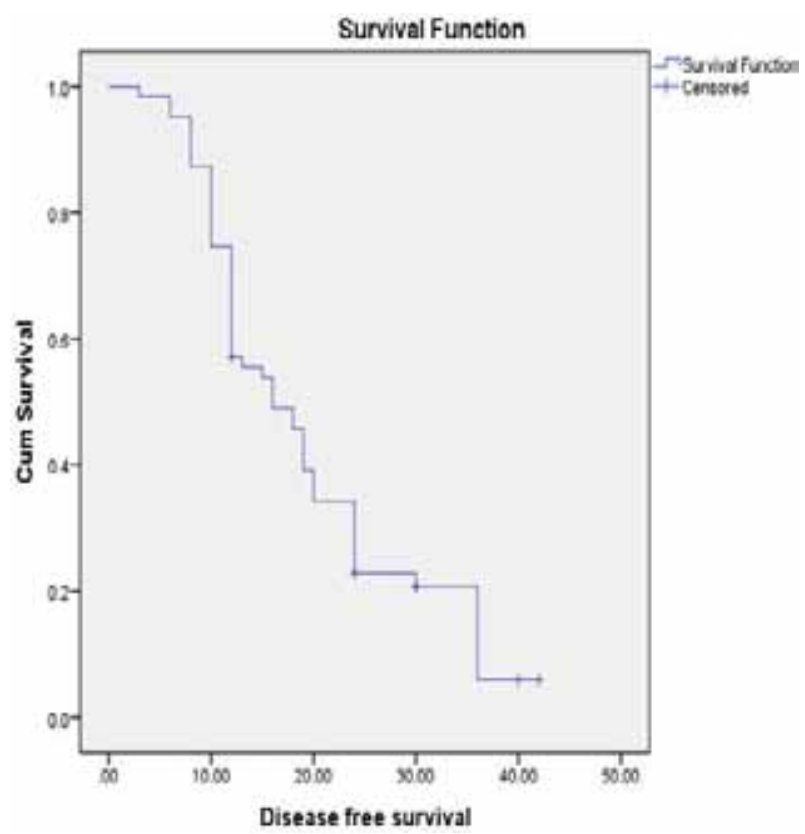

Figure 2: DFS for all molecular subtypes before HM. 
Kasr-El-Aini Journal Of Clinical Oncology And Nuclear Medicine

Vol. 10 | No. 1-2 2014

E. A. Elkhouly et al.

Table 1: Clinical Characteristics and Survival in different Molecular Subtypes

\begin{tabular}{|c|c|c|c|c|c|}
\hline Group & Patient No & $\begin{array}{c}\text { Mean age at } \\
\text { initial diagnosis }\end{array}$ & DFS (months) & $\begin{array}{c}\text { Interval from initial } \\
\text { diagnosis to } \mathrm{HM}(\text { months) }\end{array}$ & $\begin{array}{c}\text { Median Overall } \\
\text { survival (months) }\end{array}$ \\
\hline Luminal A & 33 & 46.93 & 16.15 & 21.91 & 33.27 \\
\hline Luminal B & 8 & 45.25 & 25.25 & 35.75 & 45.75 \\
\hline HER-2 enriched & 14 & 43.85 & 20.78 & 27.21 & 34.14 \\
\hline $\mathrm{TN}$ & 8 & 44.87 & 15.37 & 20.63 & 30.00 \\
\hline Overall & 63 & 45.78 & 18.24 & 24.68 & 34.63 \\
\hline$P$-value & & 0.79 & 0.16 & 0.48 & 0.02 \\
\hline
\end{tabular}

TN: triple negative

Table 2: Univariate and Multivariate Analysis of Clinical Characteristics and Treatment in all Molecular Subtypes

\begin{tabular}{|c|c|c|c|c|c|}
\hline Prognostic factor & Patient No. & $\begin{array}{c}\text { Median survival } \\
\text { (months) }\end{array}$ & $95 \% \mathrm{CI}$ & Univariate & $P$ Multivariate \\
\hline \multicolumn{6}{|l|}{ Age (years) } \\
\hline$>35$ & 10 & 32 & $25.80-38.20$ & \multirow{2}{*}{0.443} & \multirow{2}{*}{0.52} \\
\hline$\leq 35$ & 53 & 36 & $34.05-37.94$ & & \\
\hline \multicolumn{6}{|l|}{ Menstrual status } \\
\hline Premenopausal & 39 & 33 & $26.97-39.02$ & \multirow{2}{*}{0.263} & \multirow{2}{*}{0.89} \\
\hline postmenopausal & 24 & 36 & $32.93-39.07$ & & \\
\hline \multicolumn{6}{|c|}{ Disease Stage (at presentation) } \\
\hline II & 26 & 40 & $30.83-49.16$ & \multirow[b]{2}{*}{0.007} & \multirow[b]{2}{*}{0.02} \\
\hline III & 37 & 33 & $30.18-35.81$ & & \\
\hline \multicolumn{6}{|l|}{ Subtype : } \\
\hline Luminal A & 33 & 33.27 & $21.95-30.05$ & \multirow{4}{*}{0.012} & \multirow{4}{*}{0.11} \\
\hline Luminal B & 8 & 45.75 & $39.06-56.94$ & & \\
\hline HER-2 enriched & 14 & 34.14 & $23.81-48.19$ & & \\
\hline $\mathrm{TN}$ & 8 & 30 & 20.91-43.09 & & \\
\hline \multicolumn{6}{|l|}{ Number of HM } \\
\hline Isolated & 25 & 40 & $30.82-49.18$ & \multirow{2}{*}{0.001} & \multirow{2}{*}{0.043} \\
\hline Multiple & 38 & 32 & $24.35-39.65$ & & \\
\hline Endocrine therapy & Before & & & & \\
\hline \multicolumn{6}{|l|}{ Metastases } \\
\hline TAM & 27 & 36 & $25.99-46.01$ & \multirow{3}{*}{0.772} & \multirow{3}{*}{0.41} \\
\hline AI & 8 & 35 & $32.54-37.46$ & & \\
\hline No & 28 & 33 & $28.96-37.04$ & & \\
\hline \multicolumn{6}{|c|}{$\begin{array}{l}\text { Endocrine therapy after } \\
\text { metastases }\end{array}$} \\
\hline TAM & 27 & 16 & $0.11-3.26$ & \multirow{3}{*}{0.13} & \multirow{3}{*}{0.62} \\
\hline $\mathrm{AI}$ & 8 & 19.5 & $0.7-34.3$ & & \\
\hline No & 28 & 14 & $0.1-3.02$ & & \\
\hline \multicolumn{6}{|c|}{ Chemotherapy after metastases: } \\
\hline Yes & 50 & 17 & \multirow{2}{*}{$0.0-2.17$} & \multirow{2}{*}{$0.18 \#$} & \multirow{2}{*}{0.68} \\
\hline No & 13 & 12 & & & \\
\hline
\end{tabular}

HM: hepatic metastasis \#Fisher exact test 


\section{DISCUSSION}

Breast cancers could be classified by using complementary DNA microarrays and hierarchical clustering techniques into five molecular subtypes: luminal A, luminal B, HER2-enriched, basal-like, and normal breast-like. Because of the different clinical outcomes of subtypes of breast cancers, numerous studies had analyzed the association between breast cancer subtypes and prognosis ${ }^{14}$. Owing to limited fresh specimens and slashing technique, complementary DNA microarrays could not been used, and in our study, we used a gene expression profile-validated immunehistochemical surrogate panel to distinct subtypes of breast cancers.

Harrell, et al, had reported that HER2-enriched subtype tumors vigorously spread to the liver, while TN subtypes transfer to the brain and lung7. TN subtype has been reported previously with poor outcomes ${ }^{15}$. The present study, HER2-enriched subtype patients have a better survival time than TN subtype (median OS is 36 and 32 moths respectively). It could be due to the resistance to systemic therapy, biological characteristics of the TN subtype breast cancers, small samples numbers, and economic problems.

Generally, chemotherapy, surgery, and interventional therapy were the main treatments for patients with hepatic metastases. Endocrine therapy commonly was given to patients with positive hormonal status, long DFS, non life threatening metastases, and better performance status. The present study detects the effect of endocrine therapy on luminal A and luminal B. Because none of HER2-enriched subtype patients received Anti-HER2 (Herceptin) due to departmental, governmental policy and limited resources at that time of diagnosis, we could not explore endocrine therapy was a prognostic factor or not for this subtype of patients. Because of non triple negative group of patients receiving endocrine therapy, endocrine therapy could not be explored as a prognostic factor for TN subtype patients or not. Luminal A group of patients treated with endocrine therapy did improve more than patients treated without endocrine therapy (median OS of 35 vs. 30 months, $P=0.19$ ), Figure 8 . Similarly, a better tendency of median OS for luminal B patients treated with endocrine therapy was found to be more than patients not treated with endocrine therapy after $\mathrm{HM}$ (median OS is 48 months Vs 40 months, $\mathrm{P}=0.13$ ), figure 9. Explanation of this observation might be due to genetically and biologically heterogeneity between primary tumor of breast cancer and HM from breast cancer, luminal B is not one group some are HER2 positive and some not, it would be useful to obtain liver biopsy and analyze for molecular profile transformation if possible. Prospective study with large samples and all treatment facilities to validate the relation of HER2-enriched breast cancer with HM and endocrine therapy is to be expected.

The clinico-pathologic characteristics of the primary tumor of patients with HM may influence the outcome of patients. In the present study, age was not an independent prognostic factor. However, age less than 35 years had a tendency of a short survival time after HM. It could be due to the fact that there is an association of tumor high propensity of proliferation, intravasation, and angiogenesis with young age at diagnosis. Univariate analysis for survival after HM in all molecular subtypes showed that: disease stages at presentation, molecular subtype, and number of HM are significant prognostic factors (Table 2).

The results of the present study in the above point were opposite to the results quoted by Qi-Dong Ge, et al. In his study, analyses were performed between patients, who received adjuvant endocrine therapy before hepatic metastases diagnosis, and patients, who did not received endocrine therapy. The results showed endocrine therapy was not a significantly favorable prognostic factor for luminal subtype patients, who were initially treated with endocrine therapy $(P=0.4) 20$. This may be explained by the difference in samples size.

\section{CONCLUSION}

In conclusion, the present study demonstrated that breast cancer patients with hepatic metastases have a poor prognosis. Multivariate analysis showed that: advanced stage, non luminal subtypes, and multiple HM were unfavorable independent prognostic factors. Endocrine therapy can improve the survival time and appear to be a reasonable treatment for luminal subtypes patients with hepatic metastases from breast cancer. The present study is limited by its small samples and retrospective design, future clinical study with large samples and a prospective design are expected to validate the hypothesis and findings.

\section{REFERENCES}

1. Shibuya K, Mathers CD, Boschi-Pinto C, Lopez AD, Murray CJ. Global and regional estimates of cancer mortality and incidence by site: II. Results for the global burden of disease 2000. BMC Cancer 2002;2:37.

2. Early Breast Cancer Trialists' Collaborative Group (EBCTCG). Effects of chemotherapy and hormonal therapy for early breast cancer on recurrence and 15-year survival: an overview of the randomised trials. Lancet 2005;365(9472):1687-717. 
3. Miller KD, Sledge GWJ. The role of chemotherapy for metastatic breast cancer. Hematol.Oncol.Clin.North Am. 1999;13(2):415-34.

4. Zinser JW, Hortobagyi GN, Buzdar AU, Smith TL, Fraschini G. Clinical course of breast cancer patients with liver metastases. J.Clin.Oncol. 1987;5(5):773-82.

5. Parker JS, Mullins M, Cheang MC, Leung S, Voduc D, Vickery T, et al. Supervised risk predictor of breast cancer based on intrinsic subtypes. J.Clin.Oncol. 2009;27(8):1160-7.

6. Cheang MC, Voduc D, Bajdik C, Leung S, McKinney S, Chia SK, et al. Basal-like breast cancer defined by five biomarkers has superior prognostic value than triplenegative phenotype. Clin.Cancer Res. 2008;14(5):1368-76.

7. Harrell JC, Prat A, Parker JS, Fan C, He X, Carey L, et al. Genomic analysis identifies unique signatures predictive of brain, lung, and liver relapse. Breast Cancer Res.Treat. 2012;132(2):523-35.

8. Bernard-Marty C, Cardoso F, Piccart MJ. Facts and controversies in systemic treatment of metastatic breast cancer. Oncologist 2004;9(6):617-32.

9. Sinn HP, Helmchen B, Wittekind CH. [TNM classification of breast cancer: Changes and comments on the 7th edition]. [Article in German]. Pathologe 2010;31(5):361-6.

10. Piñero-Madrona A, Polo-García L, Alonso-Romero JL, Salinas-Ramos J, Canteras-Jordana M, Sola-Pérez J. Immuno-histochemical Characterization of Breast Cancer: Towards a New Classification? Cir.Esp. 2008;84(3):138-45

11. Kobayashi T, Ichiba T, Sakuyama T, Arakawa Y, Nagasaki E, Aiba K, et al. Possible clinical cure of metastatic breast cancer: Lessons from our 30-year experience with oligometastatic breast cancer patients and literature review. Breast Cancer 2012;19(3):218-37.
12. Tan S, Wolff AC. Treatment of metastatic breast cancer: Chemotherapy. In: Harris JR, Lipp man ME, Morrow M, Osborne CK, editors. Diseases of the breast. 4th ed. Philadelphia: Wolters Kluwer/Lippincott Williams \& Wilkins; 2010. p. 877-919.

13. Cardoso F, Kyriakides S, Penault-Llorca F, Poortmans P, Senkus E, Thompson A, et al. Primary Breast Cancer: ESMO Clinical Practice Guidelines. Ann.Oncol. 2013;24(Suppl 6):vi7-vi23.

14. Hu Z, Fan C, Oh DS, Marron JS, He X, Qaqish BF, et al. The molecular portraits of breast tumors are conserved across microarray platforms. BMC Genomics 2006;7:96.

15. Sorlie T, Tibshirani R, Parker J, Hastie T, Marron JS, Nobel A, et al. Repeated observation of breast tumor subtypes in independent gene expression data sets. Proc. Natl.Acad.Sci.U S A. 2003;100(14):8418-23.

16. Wyld L, Gutteridge E, Pinder SE, James JJ, Chan SY, Cheung KL, et al. Prognostic factors for patients with hepatic metastases from breast cancer. Br.J.Cancer 2003;89(2):284-90.

17. Patanaphan V, Salazar OM, Risco R. Breast cancer: Metastatic patterns and their prognosis. South.Med.J. 1988;81(9):1109-12.

18. O'Reilly SM, Richards MA, Rubens RD. Liver metastases from breast cancer: the relationship between clinical, biochemical and pathological features and survival. Eur.J.Cancer 1990;26(5):574-7.

19. Hoe AL, Royle GT. Breast liver metastases-incidence, diagnosis and outcome. J.R.Soc.Med. 1991;84(12):714-6.

20. Ge QD, Lv N, Kong YN, Xie XH, He N, Xie XM, et al. Clinical characteristics and survival analysis of breast cancer molecular subtypes with hepatic metastases. Asian Pac.J.Cancer Prev. 2012;13(10):5081-6. 